\title{
Workflow Scheduling in Cloud Computing
}

\author{
Rajwinder Singh \\ Lovely Professional University \\ Jalandhar \\ Punjab
}

\author{
Pushpendra Kumar Pateriya \\ Lovely Professional University \\ Jalandhar \\ Punjab
}

\begin{abstract}
Cloud Computing offers wide computation and resource facilities for execution of various application workflows. Many different resources involved in execution of single workflow. Cloud Computing offers highly dynamic environment in which the system load and status of resource changes frequently. As the workload increases with increase in Cloud Services and clients there is a need to handle these requests or jobs. It needs to schedule them first to execute on different available VMs. The execution of cloud workflows faces many uncertain factors in allocating and scheduling workload. The first step is to provide an efficient workflow allocation model by considering the client's requirements. Workflow scheduling model will schedule jobs in such a way that all the jobs will get executed taking minimal possible time, maintain QoS and satisfy client's requirements.
\end{abstract}

KEYWORD: $\quad$ VM(Virtual Machine),Cloud Computing, Workflow, Workflow Scheduling.

\section{INTRODUCTION}

Cloud Computing is a large-scale distributed computing paradigm that is driven by economies of scale, in which a pool of abstracted virtualized, dynamically-scalable, managed computing power, storage, platforms, and services are delivered on demand to external customers over the Internet [1]. Cloud Computing is the use of computing resources (hardware and software) that are delivered as a service over a network [2]. Cloud computing is a latest new computing paradigm where applications, data and IT services are provided over the Internet. The on-demand, self-service, payby-use nature of cloud computing is also an extension of established trends. From an enterprise perspective, the ondemand nature of cloud computing helps to support, the performance and capacity aspects of service-level objectives. The self-service nature of Cloud Computing allows organizations to create elastic environments that expand and contract based on the workload and target performance parameters. And the pay-by-use nature of Cloud Computing may take the form of equipment leases that guarantee a minimum level of service from a Cloud provider [3].

Workflow is defined as the automation of a business process, in whole or in part, during which documents, information or tasks are passed from one participant to another for action, according to a set of procedural rules [4]. When talk about cloud, the major advantage of cloud is its application scalability or elasticity. This elastic nature of cloud facilitates changes of resource and characteristics at run time. This ability of cloud enables the workflow management systems to readily meet the quality of service requirements of application [5].

The WfMC (Workflow Management Coalition) defines workflow as [4], identifying the interfaces within this structure which enable products to interoperate at a variety of levels. This model defines a workflow management system and the most important system interfaces. The below figure shows the reference model of workflow as:

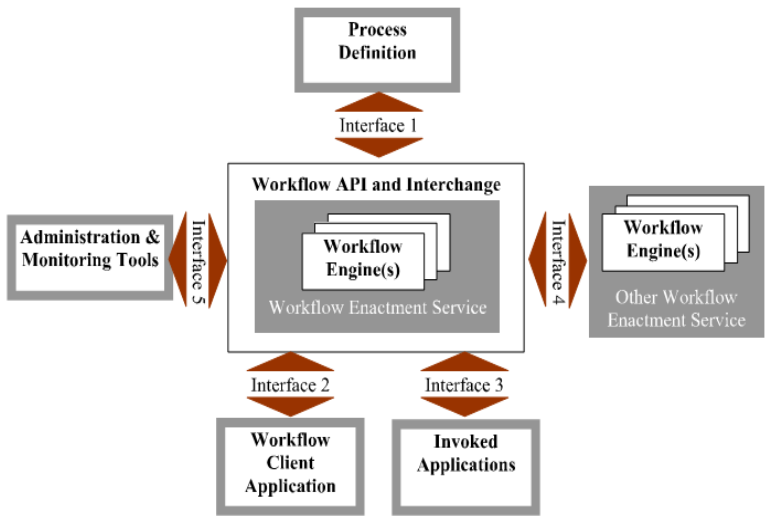

Figure 1: Workflow Reference model [4]

Workflow engine: Software that provides the run-time environment in order to create, manage and execute workflow instances.

Process Definition: The representation of a workflow process in a form which supports automated manipulation.

Workflow Interoperability: Interfaces to support interoperability between different workflow systems.

Invoked Application: Interfaces to support interaction with a variety of IT applications.

Workflow Client Application: Interfaces to support interaction with the user interface.

Administration and Monitoring: Interfaces to provide system monitoring and metric functions to facilitate the management of composite workflow application environments [4].

\section{RELATED WORK}

There are various workflow scheduling models developed by number of researchers. Some of them are explained as:

\section{POSEC and Pareto Analysis}

Especially in cloud, when to talk about Job mapping and Job mapping and scheduling, there are two algorithm which works on these jobs for the optimization scheduling: one is algorithm based on POSEC method [6] and the other is algorithm based on Pareto analysis. POSEC is Prioritize by Organize, Streamlining, Economizing and Contributing. The POSEC method prioritizes the jobs on the basis of their parameters like organize, streamlining, economizing and contributing and then categorize the jobs into four level keeping its urgency and importance as parameter. The scheduling of jobs is then applied on these levels for optimal execution.

According to Pareto Analysis[6] 80\% of tasks completes its execution in $20 \%$ of time and rest $20 \%$ jobs can execute in rest $80 \%$ of time. If the higher priority jobs are put into first $80 \%$ jobs category then the execution of jobs takes very less time as the important or prioritized jobs will execute first and thus model build is more optimal. It has been found that 
algorithm based on Pareto Analysis take less time to execute the same set of jobs as executed using algorithm based on POSEC method [7]

\section{Hierarchical cloud workflow scheduling schema}

Another way to achieve optimal schedule is by using hierarchical cloud workflow scheduling schema. According to this schema the whole workflow scheduling is divided into three stages: at very first stage whatever parallel job requests are coming, it splits all the parallel jobs. During its second step matching of jobs with corresponding candidate services takes place. This means the resources are assigned to different jobs as per their requirements. And in the third/last stage a scheduling algorithm is applied for the execution of these jobs. Using Heuristic Generic algorithm this scheme can achieve an optimal workflow schedule [8].

\section{Iterative Ordinal Optimization}

Iterative Ordinal Optimization is another method of achieving an optimal workflow scheduling in cloud computing environment. The advantage of using this method is it lowered overhead in producing suboptimal schedule and ability to work well on elastic cloud under dynamic workload [9].

\section{One-port model and Multi-port model}

Specifically for the linear workflow, a linear workflow is what in which dependencies between stages can be represented by a linear graph, and to schedule such workflow two methods are used: one-port model and multiport model. One-port model is one in which each processor can perform computation, receiving incoming task or sending output one at a time only. There is no parallel processing in one-port model but in case of multiport model a processor can perform multiple operations like computation, receiving input etc at one time. Multiport model allows multiple incoming and outgoing at the same time. These two algorithmic models are useful for linear workflow optimization and helps in minimizing the latency [10]. This will lead to an optimal workflow scheduling for linear workflow.

\section{Activity based costing in cloud computing}

Activity based costing in cloud computing is another model for optimal workflow scheduling. This Activity based costing is the way to measure both cost of the object and its performance. According to Activity based costing model, a task can be evaluated separately on the bases of their resources, space and time taken to completely execute. A job can be categorized on the basis of Available and Partially Available factor. An Available job is one whose all required resources for execution is available at same data center only and a Partially Available job is such whose required resources for its execution is not present on single data center which means resources are scattered among different data centers. For a Partially Available job resources need to be collect from different data centers for its execution. The jobs are further subdivided on the basis of their dependencies as Dependent and Independent jobs [11]. The scheduling can be done at this bottom level for the optimality of workflow scheduling.

Two levels task scheduling mechanism based on load balancing

Two levels task scheduling mechanism based on load balancing is one way for task scheduling optimization in cloud computing system. According to this method tasks are scheduled at scheduling optimizer and this scheduling optimizer take information from system model and predicted execution time model which keep track of all the resources and predicted execution information. Scheduling optimizer itself checks whether if the current prepared scheduled is optimize or not if not it will regenerate the optimal schedule [12]. Using this model the execution time decreases and also the resource utilization increases.

\section{PROBLEM FORMULATION}

A cloud computing is a very big network where the millions of users accessing thousands of servers all times. Scheduling the tasks/Jobs at the server end is very difficult for a server, because the number Job requesting is very large in number, which required some resources to execute. The schedule thus build by the server must be optimal and good enough so that each request by the user gets response in time, and every Task/Job gets proper resources for its execution.

In this paper there is a proposed model for Scheduling workflow. The Workflow Scheduling model thus builds will be optimal which means the Job/Task will take the minimum time for its execution and will get the reliable resource on time.

\section{PROPOSED MODEL}

A cloud computing is a very big network where the millions of users accessing thousands of servers all times. Scheduling the tasks/Jobs at the server end is very difficult for a server, because the number Job requesting is very large in number, which required some resources to execute. The schedule thus build by the server must be optimal and good enough so that each request by the user gets response in time, and every Task/Job gets proper resources for its execution.

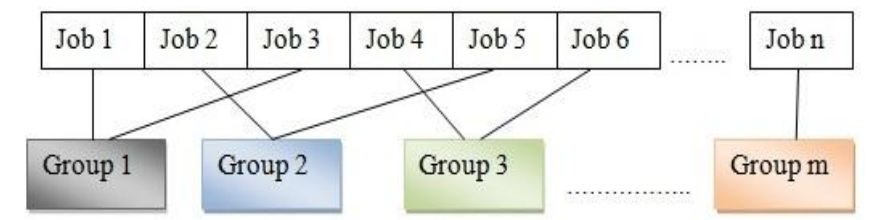

Prioritizing Jobs inside Groups

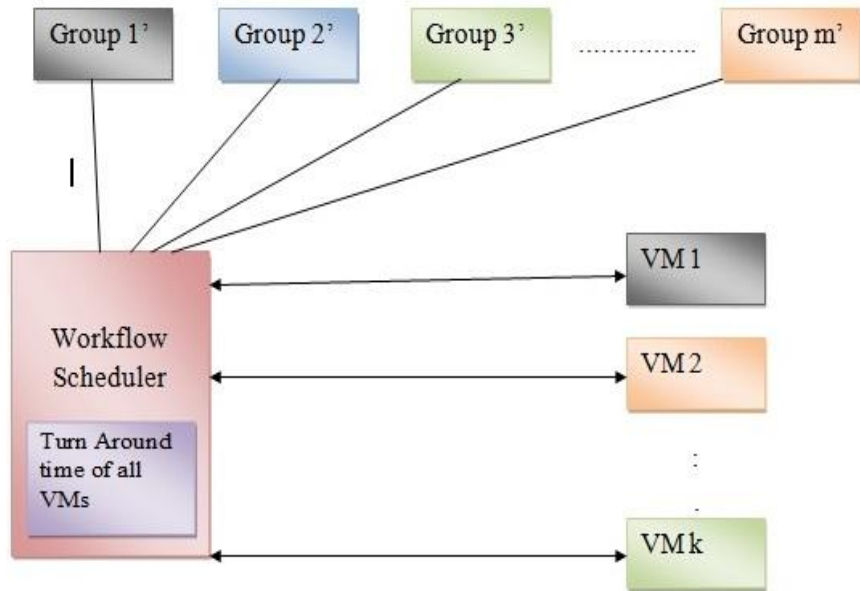

Figure 2: Model explaining grouping of jobs and scheduling

When to talk about Cloud, there are different servers over the cloud which takes client's requests and after execution gives respond to the clients. Most of these servers are built on different Virtual Machines (VM) or in other words these Virtual machines take input from clients and after execution respond back. Taking consideration about the servers as VMs and these VMs 
respond to client's requests. For the optimal Workflow Scheduling for such system, proposed model is given as:

- Step 1: All the incoming tasks will be grouped together as according to their certain behavior or attribute (like deadline constrained or low cost requirement).

- Step 2: After grouping of task, the tasks will be prioritized. A task can be prioritize on the basis of its attributes like deadline, execution time etc.

- Step 3: The Virtual machine with minimum response time and is capable to execute selected task will be assigned for the execution of the task.

\section{CONCLUSION AND FUTURE WORK}

This paper involves an overview of Workflow execution in Cloud Computing and Workflow Scheduling in Cloud Computing. The main idea for proposing this model is to improve the cost based on QoS requirement and grouping of resources and jobs over the cloud. Thus proposed workflow scheduling model will optimize the Turnaround time for the whole process. For the next part of this research, we are going to implement this proposed model on Cloudsim (a framework for modeling and simulation of Cloud Computing).

\section{ACKNOWLEGDEMENT:}

In all humility and with much fervor, I owe my deep and sincere gratitude to Assistant Prof. Harshpreet Singh of Computer Science Engineering, LPU, Jalandhar, India for his enlightened guidance, continuous encouragement, esteemed supervision, and paternal affection throughout the period of this research. Key improvements in the proposed research work would not be possible without the valuable suggestion and the feedback of my guide.

\section{REFRENCES}

[1]. Twenty-one Experts Define Cloud Computing. (2008). Retrieved october 2012, from SYS-CON Mdeia Inc: http://cloudcomputing.sys-con.com/read/612375_p.htm
[2]. Cloud Computing. Retrieved september 2012, from en.wikipedia.org:

http://en.wikipedia.org/wiki/Cloud_computing

[3]. Sun Microsystems, I. (2006). Introduction to cloud computing architecture.

[4]. liu, k. (1999). Workflow Management Coalition, Workflow Management Coalition Terminology \& Glossary.

[5]. S. Pandey, D. K. Cloud Computing: Principles and paradigms. Wiley STM.

[6]. Time management. (n.d.). Retrieved september 2012, from http://en.wikipedia.org/wiki/Time_management

[7]. Navjot Kaur, T. S. (2011). Comparison of workflow Scheduling Algorithms in Cloud Computing. Internation Journal of Advanced computer Science and Applications , 2-10.

[8]. Cheng, B. (2012). Hierarchical Cloud Services Workflow scheduling Optimization schema using Heuristic Generic Algorithm. ISSN .

[9]. Fan Zhang, J. C. (n.d.). Ordinal Optimized scheduling of scientific workflow in Elastic Compute Clouds.

[10].K. Agarwal, A. B. (2010). Scheduling Algorithms for linear workflow optimization. IEEE .

[11].Ashutosh Ingole, S. C. (2011). An optimized Algorithm for task scheduling based on activity based costing in Cloud Computing. International Journal of Computer Applications(IJCA) .

[12].Tayal, S. (2011). Tasks Scheduling Optimization for the Cloud Computing System. International Journal of Advanced Engineering Science and Technologies (IJAEST), 111-115. 